\title{
Lockdowns and Suppression: Was it? Will it have been worth it?
}

\author{
Dr Peter G Grove ${ }^{1}$ \\ Independent Researcher \\ peter.g.grove@btinternet.com \\ Version $10-20^{\text {th }}$ January 2021
}

1. Former Chair UK Department of Health's economic/analytical Senior Review Committee (IASRC) and also SPI-M. Member of SAGE in the H1N1 pandemic and in the Ebola crisis.

\section{Introduction}

In normal, non-Covid times, if you live in one of the most deprived areas of the UK your life expectancy will be smaller than those living in the most affluent by a little less than 10 years $^{1}$. The average age of those dying from Covid-19 in the UK generally is 82 years and thus the reduction in life years is also a little less than 10 years. However even at an age of 82 there is less than $10 \%$ risk of dying if infected. In addition, even in a reasonable worst case scenario there is only about $1 / 3$ risk of being infected. The overall risk of losing the 10 years of life due to Covid-19 for eighty-odd year olds is hence $\sim 3 \%$. On the other hand there is a 10\% 'risk' of living in a heavily deprived area, Covid or no Covid, with a similar reduction of life expectancy. Despite the very significantly greater ongoing risk of simply living in a deprived area than that from Covid-19, the Government will spend an additional $£ 380$ billion pounds this year on Covid-19 - a significant portion of GDP. On the other hand over the last decade deprivation has at best remained at a constant level given the 'need' to reduce Government debt by 'austerity'.

This is a very 'back of the envelope' calculation of the relative impact of Covid and deprivation, defective in many ways - but it indicates the need to carry out an analysis to establish reasonable priorities in Government spending.

Such an analysis is required in all UK Government Departments in the normal run of business. It is called an Impact Analysis or IA.

In the Spring I put a Freedom of Information request into the Department Of Health and Social Care for the Impact Assessment supporting the change of policy to suppression (i.e. intermittent lockdown separated by periods of lesser controls) from mitigation (the decades old pandemic policy).

The Department has belatedly replied saying no Impact Analysis has been carried out in the many months since the crisis began.

\footnotetext{
${ }^{1}$ https://www.health.org.uk/blogs/the-healthy-life-expectancygap?gclid=CjwKCAiAz4b_BRBbEiwA5XIVVux18ZkV1eYH1dWnBrmeZCMK4onZqqSG9RgqMFCkXkUMA8HjtFdILR oCZAsQAvD_BwE
} 
I have thus carried out my own Impact Assessment following Government practice (the Treasury Green Book). You will see that even if one makes very favourable assumptions for the lockdown policy, the original mitigation policy is considerably superior.

It also indicates that an economically rational decision maker would have chosen the mitigation strategy, over 'lockdown' even in March $2020^{2}$.

It is important to understand that the analysis is not 'wealth v. health' but in terms of 'social value', an attempt to formalize how much society values aspects of everyday life based on their behaviour. If the resources had been used elsewhere (e.g. to reduce deprivation) they could have saved lives - by taking them from the economy they will lead to deaths.

There are clear limitations to this approach but it is how spending decisions are supposed to be made in Government.

One example is that, following Government practice, the IA ignores the additional international effects of the suppression policy such as children going hungry in Bangladesh due to the drop in UK economic activity ${ }^{3}$.

My own view is that, however deficient a purely UK focussed utilitarian analysis of the kind presented here, the eventual historical narrative of 2020 lockdowns will be that the poor, the young are paying the price now for protecting the older, wealthier population and will be made to pay the price in the coming austerity for a generation.

Whatever the case, the formal analysis the Government could have carried out in March 2020 follows.

Of course, having made an economically irrational choice of policy in March, it has been difficult to retrieve the situation. The current situation is considerably more complicated than that considered in the IA. It is important to realise that this complexity is a consequence of the policy not the disease.

The complexity is (fortunately) further increased by the arrival of the vaccines; the above analysis indicates the importance of carrying out impact assessments on various policy options for removing restrictions as vaccination protection increases - in particular when protection is achieved for the most vulnerable groups.

\footnotetext{
'The Assessment above does not consider the 'elimination' policy suggested by some such as 'Independent SAGE'. See Notes for discussion.

${ }^{3}$ See https://www.bbc.co.uk/news/world-asia-52417822
} 


\title{
HIGH LEVEL IMPACT ASSESMENT FOR 2020/21 CORONAVIRUS EPIDEMIC
}

\section{Was the UK Government's decision to move from the longstanding mitigation to 'lockdown' or suppression policy economically rational?}

\author{
Dr Peter G Grove ${ }^{4}$
}

\section{Summary}

The UK Government has not carried out an Impact Assessment of its Coronavirus policies. Such assessments are a normal part of Government policy formation and Ministers are expected to personally sign off policies as cost beneficial.

This short paper considers a high level assessment of the kind that normally would have been produced (and could have been produced rapidly) before the introduction of the suppression or 'lockdown' policy on the $23^{\text {rd }}$ March. The issue at question is the relative benefits of the mitigation policy, which was the standing Government pandemic policy until March 2020, compared with those of the 'lockdown' or suppression policy actually adopted in that month. It shows that all unquantifiable and the net quantifiable benefits favour the abandoned mitigation policy.

Further it shows that an economically rational decision maker would have chosen the mitigation strategy, over' lockdown' even in March 2020.

\section{Scenarios considered:}

Mitigation Policy (pre-lockdown strategy) : a mitigated epidemic until vaccine introduction in March 2021. The scenario generally follows the 'mitigation' scenario of the famous Imperial modelling paper ${ }^{5}$. The main variation from the scenario is the use of a number of less pessimistic infection fatality estimates as discussed in detail below. This is not to say that we believe this scenario represents the best that could be done in terms of mitigation (for example effective shielding of the vulnerable) but it provides a conservative (see below) starting point. In this scenario the epidemic would have essentially been over by the beginning of September 2020 with contact tracing maintaining low case levels until March 2021. (Informal social distance measures, hand hygiene etc. may also have been required in the interim pre-vaccine period).

'Lockdown' policy or suppression policy as actually implemented by Government: This is the policy currently being implemented with a first and second wave (taken as ending in the December lockdown). It follows the Imperial suppression scenario complete with circuit breaker lockdowns as described in the Imperial paper, and assumes minimal third (fourth, fifth....) wave deaths. By 'conservative' assumptions I will mean assumptions that favour this scenario. The low level of deaths in later waves is an example of such a conservative assumption.

\footnotetext{
${ }^{4}$ Sometime Chair, UK Department of Health's economic/analytical SENIOR REVIEW COMMITEE (IASRC)

5 Impact of non-pharmaceutical interventions (NPIs) to reduce COVID-19 mortality and healthcare demand - March 2020 https://www.imperial.ac.uk/media/imperial-college/medicine/sph/ide/gida-fellowships/Imperial-CollegeCOVID19-NPI-modelling-16-03-2020.pdf
} 


\section{Why chose a scenario cut off date of March 2021?}

1. Doubts have been expressed about long term immunity from natural infection. There is negative evidence from declining antibodies and positive evidence from significant T-cell responses out to six months. Whatever the case however, there is no real evidence of significant re-infection and thus even the most pessimistic view is that there will be little repeat infection before March. We will thus follow the Government (SPI-M-O) modellers in assuming immunity in the short term until March. This is a conservative assumption (in the sense outlined above). If as is probable significant long term immunity is achieved, then the benefit of the mitigation scenario would be increased by the reduced need for vaccines. Equally it is unlikely that, even with good evidence for natural herd immunity, a vaccination programme would not be implemented given the existence of a working vaccine.

2. Government financial plans (such as borrowing and the furlough) have been set out to March in detail.

\section{Infection fatality rate}

Realistic estimates of the infection fatality rate (IFR) range from $0.1 \%$ to $1 \%$. For example, the base estimates of CDC in the United States have varied from $0.3 \%$ to $0.7 \%$ but with a credible intervals ranging up to $1 \%$. In part the variation of estimates is due to methodology - those based on severe symptomatic cases being higher than those from serology which are in turn higher than those from following traced infections. There is also a considerable problem of definition given the 1000 fold variation in risk of death following infection with age. An average figure is thus highly dependent on the population to which it is applied. The average in the Imperial modelling for the UK was chosen to be around $1 \%$.

Serology (ONS) $)^{6}$ suggests that $5-10 \%$ of the population were infected in the first wave. Given the $\sim 45,000$ deaths we obtain a population average IFR of $\sim 1 \%$. We take this as our highest IFR estimate. Information from the King's College symptom tracker, however, suggests that either only one half of those infected seroconvert or possibly half of those infected lose their antibodies too quickly to be surveyed ${ }^{7}$. Twice as many were therefore infected as the serology suggests ${ }^{8}$. Hence we assume a lowest reasonable IFR of $0.5 \%$ with our mortality estimates suitably adjusted. We also consider an intermediate IFR of $0.7 \%$, which is also around the middle of the range considered by SPI-M/SAGE ${ }^{9}$. The value of $0.7 \%$ will be taken as the base case for exposition but the results for all three IFRs $(0.5 \%, 0.7 \%$ and $1 \%)$ will be presented.

\section{Quantified Net Benefits of the lockdown policy over the mitigation policy}

The main benefit of the lockdown policy against the mitigation scenario is in saving lives and reducing the number of serious complications. The mitigation scenario was calculated to cost

\footnotetext{
${ }^{6}$ https://www.ons.gov.uk/peoplepopulationandcommunity/healthandsocialcare/conditionsanddiseases/bulleti ns/coronaviruscovid19infectionsurveypilot/23october2020\#antibody-data-for-england

${ }^{7}$ https://covid.joinzoe.com/post/covid-antibodies

${ }^{8}$ Results consistent with Italian T-cell testing https://www.medrxiv.org/content/10.1101/2020.11.09.20228023v1

${ }^{9}$ https://www.gov.uk/government/publications/spi-m-o-covid-19-reasonable-worst-case-planning-scenario-

21-may-2020
} 
$\sim 250,000$ lives. As we have seen this is a likely overestimate due to the inflated IFR. Using an IFR of $0.7 \%$ gives 175,000 deaths. Deaths under the lockdown scenario are already in excess of $65,000^{10}$. The benefit in terms of lives saved is thus 110,000.

It is important to remember that we are using the IFR adjusted Imperial mitigation policy as a worst case or conservative assumption. Swedish practice from the first wave, for example, shows that this might be greatly reduced. Indeed taking Swedish experience as directly transferable to the UK would suggest more lives would be saved under the mitigation policy-although this would require the longer imposition of extensive social distance measures.

To value these lives we must estimate the social value of a covid death following UK Government guidance (The Green Book ${ }^{11}$ ). We will triangulate a value based on three approaches:

1. Willingness to pay to prevent a road fatality. DfT estimated this ${ }^{12}$ at $f 1.25$ million in 2012. Adjusting for inflation this comes to $\sim f 1.5$ million. Some would argue that this should be increased by a measure of lost output. Department of Health practice however, is not to take this into account in general as over a lifetime as consumption broadly balances (if not exceeds) production.

2. The Green Book value a Quality Adjusted Life Year at $£ 60,000^{13}$. In general, Quality of Life does not exceed 0.85 for those over $45 y$ years, that is those at risk of death from covid. If we use Government standard 3.5\% discounting we can largely ignore the lesser life expectancies of those over 45 (a conservative assumption). We obtain a discounted value of a life as $(£ 60,000 * 0.85 / 0.035)=\sim £ 1.5$ million.

3. Some argue that health should be discounted at $1.5 \%$ per year rather than $3.5 \%$, We disagree with this for technical reasons too involved to discuss here. However if we use the lesser discounting rate the life expectancy of those who die becomes important. Given most deaths are in those over 70, we make the assumption that life expectancy is of the order of 30 years and to be consistent we use the QoL for that age group of 0.75 . This gives us a little less than $f 1.5$ million.

The triangulation suggests that $£ 1.5$ million $^{14}$ is a conservative estimate of the social valuation of a lost life due to Covid-19.

The 110,000 saved deaths, thus translate into a $£ 165$ billion social value saving ${ }^{15}$.

In addition there will be a significant saving from prevention of 'long term' Covid, which we will define here as disease lasting more than 4 weeks but in a small number of cases up to 6 months or

\footnotetext{
${ }^{10}$ December 2020

${ }^{11}$ https://www.gov.uk/government/publications/the-green-book-appraisal-and-evaluation-in-centralgovernent

${ }^{12}$ https://assets.publishing.service.gov.uk/government/uploads/system/uploads/attachment_data/file/254720 /rrcgb-valuation-methodology.pdf

${ }^{13}$ Not to be confused with the $£ 20,000-£ 30,000$ cost per QALY used by NICE. The $f$ measure in this figure refers to NHS expenditure. Each $£ 1$ of NHS expenditure is considered to buy $£ 2$ to $£ 3$ of social value.

${ }^{14}$ See 'notes section' at end of paper.

${ }^{15}$ It is not clear that the treatment costs of those who would have been hospitalised should also be counted here as they would have been simply taking up NHS resource which was, in fact, lost by being unused during lockdown - due to the shutdown of non-Covid treatment. In any case they would be around f1-2billion in total and are essentially lost in the rounding.
} 
more. In the annex we use results Kings College symptom survey and the ONS, together with Quality of Life estimates from ME/CFS, to estimate the QALY loss due to 'long Covid'.

The $\sim 5$ million cases of long Covid saved leads to a social value saving of $\sim 20$ billion ${ }^{16}$.

The total partial quantified benefit of the lockdown policy at an IFR of $0.7 \%$ is around $\mp 185$ billion of social value. [This is not the net benefit - see below]

If we assume IFRs of $0.5 \%$ and $1 \%$, we obtain $£ 110$ billion and $\sim £ 300$ billion in social value, respectively.

Note that these are conservative estimates in the sense they favour the lockdown strategy by over estimating the benefit by ignoring deaths from the third (and any subsequent) waves generated by the lockdown approach.

\section{Quantified benefits of the Mitigation policy compared with lockdown}

The lockdown strategy has long term economic impacts. These are likely to last for decades and are unpredictable. What we do know if that there has been large amounts of Government expenditure to mitigate the effects of the lockdown. This expenditure has been financed by Government borrowing and Bank of England Quantitative Easing (buying Government debt). We can assume that the Treasury believe that each $£ 1$ borrowed and spent buys at least $£ 1$ of social value - probably much more - compensating the social value loss in the wider economy. Thus a lower limit on the social value lost in the lockdown strategy is the increase in Government borrowing until March next year.

Again this can be estimated in a number of ways (given the Government borrows even in normal times).

For example:

1. Total Bank of England Quantitative easing of $£ 450$ billion this year.

2. Estimated Government additional borrowing over $2020 / 21$ of $£ 340$ billion ${ }^{17}$ followed by $£ 40$ billion in 2021/22 (Office of Budget responsibility Forecast, November). A total of $£ 380$ billion.

A direct estimate can be made from forecasts of GDP:

3. IFS forecasts of GDP (similar to November OBR forecasts). The two wave prediction is for $\sim f 650$ billion over 5 years $\stackrel{18}{ }$.

In our usual conservative spirit we will take the lowest figure.

\footnotetext{
${ }^{16}$ We also might expect 50 to 200 thousand QALY loss from short term covid symptoms but this is not a major contribution - and is exceeded by a lockdown reduction in the quality of life of the entire population of considerably less than $1 \%$.

${ }^{17} \mathrm{https}$ ://obr.uk/efo/economic-and-fiscal-outlook-november-2020/

${ }^{18} \mathrm{https} / / /$ ifs.org.uk/uploads/CH2-IFS-Green-Budget-2020-UK-Economic-Outlook-.pdf
} 
The total quantified partial benefits of the Mitigation policy over the lockdown policy are thus in excess of $£ 380$ billion of social value. [This is not the net benefit - see below]

Before comparing the quantitative benefits we will consider the non-quantified benefits of each policy.

Non-quantified benefits of Mitigation policy compared with lockdown

Health and Wellbeing

The lockdown policy produces mental and physical health problems due to lack of social contact and exercise these lead directly to some deaths (e.g. suicides) and poorer health.

Both policies lead to reductions in treatment for non-covid disease by the NHS. There have been attempts to estimate this (e.g. by SAGE) however existing estimates make it difficult to separate impact due to covid cases using up capacity and the impact of lockdown measures. The important fact to consider however, is that the mitigation policy only restricts NHS capacity for six months rather than twelve for the lockdown policy. Clearly the lockdown policy generates a larger loss of social value than the mitigation policy.

\section{Culture}

The lockdown policy has greatly reduced cultural activity. This may have happened temporarily under the mitigation policy but again this would only have lasted six months rather than a year.

Freedom and Human rights

The lock down policy has required large scale reductions in public freedom.

In all these areas the benefits are unquantifiable but favour the mitigation strategy.

Which policy is net beneficial?

In terms of the quantifiable benefits the mitigation has a greater social value of in excess of $£ 195$ billion ( $£ 380$ - $£ 185)$ billion at an IFR of $0.7 \%$.

At the upper credible IFR of 1\%, the most conservative estimate of the easily quantifiable benefits of the abandoned mitigation strategy still exceed by ( $₫ 380$ billion- $£ 300$ billion) $\sim$ f80billion the most optimistic estimates of the novel lockdown policy.

At an IFR of $0.5 \%$ the result is $\sim 270$ billion for the net quantitative benefits.

In all those areas with un-quantified benefits, these benefits also favour the mitigation strategy. The mitigation policy is thus economically preferred over suppression. 
Hindsight however, is a wonderful thing. If we place ourselves in the position of the policy makers in March what would be different? One difference is that it would be reasonable to take the IFR as $1 \%$ rather than $0.7 \%$ or $0.5 \%$. Another is that we would need to estimate the covid mortality from the lockdown policy. The Imperial modelling suggested this might be as low as 20,000 (or even possibly 10,000 ) but this would require the severest measures to be in place 85 to $95 \%$ of the time until March 2021. This could not have been considered a reasonable policy even in March. An expected death toll of $\sim 50,000$ would be a more reasonable estimate for more practical long term policies with longer gaps between short circuit breakers. Finally we would need a borrowing estimate. The earlier OBR forecast was for $£ 320$ billion additional borrowing but did not consider the expected second wave lockdown/circuit breaker from the Imperial paper. Allowing, as it turns out conservatively, $£ 10$ billion for the second lockdown brings us to $£ 330$ billion.

In this case the cost benefit calculation is that the most conservative estimate of the easily quantifiable benefits of the abandoned mitigation strategy and most optimistic estimates of the novel lockdown policy essentially balance. All the unquantifiable benefits however, as well as the uncertainty in the quantified benefits, favour the mitigation strategy.

Thus it is reasonable to conclude that a rational decision maker would have chosen the mitigation strategy, hopefully with some improvements of the shielding of the elderly, over the lockdown one in March 2020.

\section{Conclusion}

This short paper considered a high level estimate of the relevant benefits of the mitigation policy, which was Government's pandemic policy until March 2020, compared with those of the 'lockdown' policy actually adopted in that month. It shows that all unquantifiable and the net quantifiable benefits favour the abandoned mitigation policy.

A much more detailed Impact Assessment is required. However, Fol requests to the Department of Health and Social Care, have revealed that no such analysis, detailed or otherwise, has been undertaken ${ }^{19}$.

That said, the additional detail is unlikely to change the conclusion that a mitigation policy was a more economically rational strategy in March 2020 than the lockdown or suppression policy. Such a conclusion should have been clear to the decision makers responsible for changing the policy from a high level (even 'back of the envelope') analysis such as the one presented here. What a more detailed analysis will do however is to partially quantify many of those losses which could not be quantified in this analysis, thus giving a more detailed picture of the losses generated by choosing to change policy direction in March. The most important of these will be the long term damage to the economy and the health impacts on non-covid disease in lockdown and related measures.

\footnotetext{
${ }^{19}$ See annex 2
} 
Notes:

- We have followed Imperial College's view of the mitigation policy with even at the lowest assumed IFR of $0.5 \%, 60,000$ additional deaths over the lockdown policy. This is a worst case scenario as Swedish experience from their first wave shows that with appropriate measures this might be reduced to close to zero. Similarly effective shielding would greatly reduce the death toll.

- 60,000 deaths is not a desirable outcome and equals more than 60,000 individual tragedies however the number needs to be put in perspective. More than 600,000 people die in the UK each year. The average age of a covid death is greater than 80 years. More importantly, if the resources used mitigating the economic damage of the lockdown were deployed on health they would have the potential of saving more deaths (see below) over the coming years. The additional death toll from the lack of NHS resources for non-covid patients may well exceed 60,000.

- It may appear that the above analysis is one of money versus health. That is not the case but it is an understandable misinterpretation for two reasons:

- The argument is phased in terms of $f$ of social value. This is a conventional measure of utility, conveying that the utility might be spent in a number of different ways. The $f$ of social value is not necessarily the same as the pound of cash in your pocket. We could have used the Quality Adjusted Life Year (QALY) as our utility measure or even potential lives saved. If so the mitigation policy would have the potential to generate an extra 3 to 4 million QALYs when compared with the lockdown policy, if the resources were spent on health - or potentially save more than 130 thousand lives even at an IFR of $0.7 \%$ and substantially more at $0.5 \%$ ( 4 to 5 million QALYs, 180 thousand lives).

- Because the Government has not produced an Impact Assessment, we are forced to measure damage to the economy by Government spending to mitigate the damage. The actual damage may well be impacts to health and lives due to, for example, cuts to future health provision due to austerity measures or the well documented health impacts of poverty.

- It is standard practice to rework impact assessments at the NICE $£ 20,000-f 30,000$ cash (rather than social) valuation of a QALY. This represents the Treasury's willingness to pay taking wider political issues into account. In this case, the argument against the lockdown policy becomes even stronger with a large net benefit even at an IFR of $1 \%$ (net benefit of in the range of $£ 200$ to $£ 300$ billion).

- In this paper, $£ 1.5$ million has been taken as a conservative social valuation of a casualty of Coronavirus. Some have argued that such a social valuation of a life for victims of the first wave with mean age 82 years of age is too high. The mean life expectancy of someone at 82 years, were they in average health, is less than 10 years giving a social value of less than $£ 450,000$. Many of those dying of Covid were in poor health and many in nursing homes. Mean nursing home survival is around two years giving a social value of less than $f 100,000$. As ever we have erred on the conservative side, possibly by a large margin.

- The mitigation strategy would have led to the worst of the pandemic being over in a few months. The suppression/lockdown policy has led to months of rising and falling infection levels. This is an optimal situation for the evolution of a more transmissible variant. This may 
have happened with additional economic damage ${ }^{20}$. This analysis shows this was an unnecessary risk.

- The mitigation strategy puts a greater strain on the NHS for a shorter period of time. It is not clear which places greater strain on the staff overall but it is not unreasonable to assume that dealing with a crisis for 12 months produces longer term fatigue and mental health issues than a one off emergency even if extended over a number of weeks.

- Similarly the shielding of the elderly and vulnerable would have only been necessary (at least at full intensity) for the period of the mitigated epidemic. The psychological impact would have therefore been much less under the mitigation rather than suppression/lockdown policy.

- The Assessment above does not consider the 'elimination' policy suggested by some such as Independent $\mathrm{SAGE}^{21}$. This is partly because such an option is not covered by the Imperial paper and was therefore not on the table when the original lockdown decision was taken. It is clear from where such policies have (e.g. New Zealand) been successful the costs of the strategy might be considerably less than those of the long term suppression policy of the kind employed in the UK. Such policies have not generally been considered suitable for use in highly connected countries such as the UK. An Impact Assessment for such a policy would have to demonstrate practicality, estimate the probability of success and the impact of failure (e.g. return to the suppression policy) as well as capturing the long term costs of the policy. Other authors are encouraged to take up the challenge.

- Finally, thank you to all those who have commented and improved this note by their suggestions.

${ }^{20}$ (https://www.bbc.co.uk/news/uk-55391289)

${ }^{21}$ https://www.independentsage.org/ 


\section{ANNEX 1: LONG COVID.}

We make use of results from https://www.medrxiv.org/content/10.1101/2020.10.19.20214494v1 and https://covid.joinzoe.com/post/long-covid and also https://www.ons.gov.uk/news/statementsandletters/theprevalenceoflongcovidsymptomsandcovid1 9complications

The King's College/Zoe results give that $\sim 15 \%$ of symptomatic cases are still ill after four weeks, $\sim 5$ $\%$ at eight and $\sim 2.3 \%$ after weeks.

This can be fitted to an exponential survival distribution with time constant 0.225 weeks $^{-1}$. The survival function suggests $~ 35 \%$ of those with symptomatic covid will fall into a notional 'long covid' sub -group although the many of these will have symptoms for less than 4 weeks.

The $\mathrm{KCL}$ analysis suggests that the distribution of illness length is long tailed (i.e. declines less rapidly than an exponential). The survival distribution can also be fitted to a 'long tailed' distribution such as: proportion surviving $=C \cdot x^{[-0.42 \cdot \ln x+0.1]}, \mathrm{x}$ being the survival time in weeks, where the form is only valid for $x>0$. $C$ is a constant. The 0.1 is selected so that the low $x$ behaviour is numerically similar to the exponential for $x>1$ week.

Both functions give similar results for the effective length of long covid illness across all of those ill that is a little less than 1.5 weeks. (Corresponding to $~ 4.5$ weeks for the notional long covid population).

On the other hand the (newer) ONS analysis suggests around 1 in 5 respondents testing positive for COVID-19 exhibit symptoms for a period of 5 weeks or longer and around 1 in 10 respondents testing positive for COVID-19 exhibit symptoms for a period of 12 weeks. Fitting a double exponential survival distribution based on our experience with the Zoe data, gives a long covid tail with time constant 0.115 weeks $^{-1}$ with again $~ 35 \%$ falling into the long covid group. The effective length of long covid illness across all of those ill that is a little more than 3 weeks. (Corresponding to $\sim 8.5$ weeks for the notional long covid population.)

In our usual conservative spirit we take the higher figure of 3 weeks.

Given the most of the longest lasting symptoms are similar to ME/CFS (https://www.prohealth.com/library/the-health-related-quality-of-life-for-patients-with-myalgicencephalomyelitis-chronic-fatigue-syndrome-me-cfs-36146) we take the ME/CFS QoL decrement of around $1 / 3$.

Given 110,000 deaths and a IFR of $0.7 \%$ this leads to a morbidity disease burden of 320,000 QALY and thus a social value loss of $\sim £ 20$ billion. 


\section{ANNEX 2:}

\section{Extract of E-mail from the Department of Health and Social Care}

Thank you for your request dated 24 November, in which you asked the Department of Health and Social Care (DHSC):

"I made this request using the Fol online form on the 18th of March - I have received no reply. Has the Department (or any of it's Agencies including NHS England or PHE) carried out a cost effectiveness or cost utility analysis of the change in Government response from the 'mitigation' strategy ( as described in the report dated 16 March 2020 by the Imperial College COVID-19 Response Team) to a 'suppression' strategy? My request includes any separate analysis of the two strategies without direct comparison. If so please supply a copy of the analysis. This will help me understand UK Government policy."

Your request has been handled under the Freedom of Information Act (FOIA).

DHSC does not hold information relevant to your request. This is because there are no documents held breaking down the analysis in the form requested, and both suppression and mitigation of COVID-19 remain important elements of the Government's response. 